\title{
What Sells on the Fake News Market? Examining the Impact of Contextualized Rhetorical Features on the Popularity of
} Fake Tweets

\author{
Ezgi Akar ${ }^{1 *}$ \\ (D) 0000-0003-2868-5091
}

Tugrul Cabir Hakyemez ${ }^{2}$

(iD) 0000-0003-0646-8950

\section{Aysun Bozanta ${ }^{2}$}

(iD) 0000-0002-1768-6278

\section{Serkan Akar ${ }^{3}$}

(i) 0000-0001-8432-5424

${ }^{1}$ University of Wisconsin-Eau Claire, USA

${ }^{2}$ Ryerson University, CANADA

${ }^{3}$ University of the Incarnate Word, USA

${ }^{*}$ Corresponding author: AKARE@uwec.edu

Citation: Akar, E., Hakyemez, T. C., Bozanta, A., \& Akar, S. (2022). What Sells on the Fake News Market? Examining the Impact of Contextualized Rhetorical Features on the Popularity of Fake Tweets. Online Journal of Communication and Media Technologies, 12(1), e202201. https://doi.org/10.30935/ojcmt/11278

\section{ARTICLE INFO}

Received: 13 Jul 2021

Accepted: 15 Sep 2021

\begin{abstract}
A fake news ecosystem is akin to a marketplace where content generators and users exchange content like sellers and buyers. The popularity of a product in this marketplace is influenced by rhetorical features (ethos, pathos, and logos), topic categories (hard news, soft news, and general news), and design motivations (political intent, fun, etc.). Therefore, the determinants of the popularity of fake news should be contextualized better to understand the spreading patterns. First, we obtained a sample from a fact-checking organization $(n=439)$. Then, we categorized tweets based on their topics and design motivation by using biaxial content analysis. Next, we proposed a rhetorical framework to organize the tweet-related indicators to develop the content's systematic characterization. Finally, we examined both the primary and interaction effects of topics, design motivations, and organized rhetorical features of tweets on popularity through a negative binomial regression. The main results revealed a positive relationship between logos-related features (i.e., the number of followers) and the popularity of the fake tweets. In addition, an exciting interaction effect indicated that fake tweets designed with political intent are nearly five times less retweeted when they contain hashtags. The research and practical implications and future directions were also discussed.
\end{abstract}

Keywords: fake news, rhetorical framework, content analysis, negative binomial regression, social media

\section{INTRODUCTION}

Fake news is defined as "fabricated information that mimics news media content in form but not in organizational process or intent" (Lazer et al., 2018, p. 1094). It includes three basic forms of information disorder: misinformation, disinformation, and mal-information. These disorders are often distinguished by the degree to which fake news is based on facts and whether it was created intentionally (Wardle \& Derakhshan, 2017). Regardless of the type, it has lately become a modern plague by spreading through online communication channels at an unprecedented pace, often causing catastrophic consequences across the

Copyright (c) 2022 by authors; licensee OJCMT. This article is an open access article distributed under the terms and conditions of the Creative Commons Attribution License (http://creativecommons.org/licenses/by/4.0/). 
world (Chew \& Eysenbach, 2010; Lazer et al., 2018; Murungi et al., 2018; Pennycook et al., 2020; Vosoughi et al., 2018). Recently, infuriated by the claimed fraud in the latest U.S. presidential election, thousands stormed Capitol Hill on January 6, 2021, intending to halt Joe Biden's inauguration as U.S. president (West, 2021). The insurrection left behind five casualties (Wise, 2021) and resulted in an impeachment trial of Trump where he stood accused of inciting an insurrection by spreading false claims about the legitimacy of the U.S. presidential election (Levine \& Gambino, 2021). Evident from the story, fake news exacerbates political polarization across society and thus undermines democracy.

Moreover, these tragic events were prevalent but, unfortunately, not limited to the political domain. During the COVID-19 pandemic, the accompanying flood of fake news has also led to irreversible damage to public health. Someone died from ingesting a fish-tank cleaning product upon reading fake news, which claimed its benefits in curing COVID-19 (Mercier, 2020). All these similar events underline the fact that fake news severely impairs society's functioning in various domains. Therefore, combatting fake news requires a contextual understanding of the factors influencing the spreading patterns of fake news in different domains.

Social networking sites have become the primary news source in many countries across the world. A recent study revealed that nearly half of the population of the following countries prefer to get their daily news from social media: South Korea (57\%), Lebanon (52\%), Argentina (51\%), Vietnam (48\%), and Turkey (45\%) (Mitchell et al., 2018). Unfortunately, the volume of fake news traversing these popular news channels has largely scaled up (Ipsos Public Affairs, 2019). More concerning is the users' indifferent attitudes towards being exposed to an increasing volume of fake news (Mitchell et al., 2019). A recent report indicated that although more than half of the U.S. online users stated that fake news is a more severe issue than terrorism, drug addiction, or racism (Mitchell et al., 2019), 23\% have shared fake news (Barthel et al., 2016). Even worse, 51\% said they sometimes created their own fake news (Mitchell et al., 2019). These results suggest the peril of an exponentially growing effect of fake news given the increasing number of users across social media platforms. Therefore, how and why the users engage with the fake content remain the key issues in the combat against fake news.

The other primary actor in the fake news ecosystem, fake news generators, act upon various motivations such as political intention, fun, and financial gain (Meel \& Vishwakarma, 2019; Zannettou et al., 2019). These motivations often manifest themselves in design characteristics and are often used to spot fake news (Enoch Pratt Free Library, n.d.). For instance, the content is expected to be shockingly speculative and vitriolic in political motivation. However, the same is not valid if the motivation is financial profit. The aim here is to increase the number of sharers by creating fake news that appeals to the public. Nevertheless, there is an additional factor that heavily influences fake news's popularity amongst social media users: topic. Having noticed differential trajectories that content popularity follows in different topic categories (e.g., politics, health, celebrities) (Vosoughi et al., 2018), fake news generators differentiate their products to garner the highest audience. While subjective content appealing to emotions may increase the "velocity" of fake news (Vasu et al., 2018, p. 4) in the politics across homophilous networks (Mihailidis \& Viotty, 2017), in the category of health and technology, a neutral polysemous content may be interpreted as more logical. It thus becomes more likely to be shared by the users. Unfortunately, extant literature failed to emphasize how the design motivation and topics contribute to fake news popularity, except in a couple of studies (e.g., Vosoughi et al., 2018). The current study fills this gap by examining the individual and interaction effects of design motivation and topic on fake news popularity.

This study used a fake news marketplace analogy. We considered users and fake news generators as having a seller-buyer relationship. Sellers (content generators) design their products (fake content) and advertise them to buyers (users) through social media platforms in this marketplace. These sellers are driven by specific motivations and adjust their content to various topic categories. On the other hand, buyers decide whether to engage with these products based on the perceived level of congruence between their existing beliefs/attitudes or enjoyment. In this market, the survival chance of fake content heavily depends on the transmission rate. Identifying the unique characteristics of surviving fake news in different design motivations and topic categories constitutes the focal point of our study. We took a novel contextualized rhetorical approach to extract these characteristics. We tested our proposed fake news marketplace analogy on one of the most popular social media platforms: Twitter. We categorized fake tweets based on their topics and presumed design motivation by using biaxial content analysis in the first step. Next, we extracted tweet- 
related features based on a rhetorical framework. Last, we examined the effects of these extracted features on fake tweets' popularity across the identified topic and motivation categories using negative binomial regression models. The practical and the methodological contributions of this study can be summarized as follows:

- Our contextualized rhetorical approach can mainly serve as (1) a framework for the characterization of fake news, (2) a guideline for automated fake news detection efforts across various topic categories and design motivations, and (3) an enhancement to social media literacy programs.

- We propose a mixed-method approach where we use an exploratory sequential design that combines qualitative and quantitative methods. The biaxial qualitative content analysis enables systematical coding and categorization of the tweets. We also quantify the tweet content by using sentiment analysis. We then use the outputs of these analyses in our negative binomial regression as inputs. This methodological approach can also be extended to research that uses textual data on other domains.

The rest of the paper is organized as follows: Section Literature Review briefly discusses the relevant literature on the fake news ecosystem and understanding fake news from a rhetorical perspective. This section is followed up by the description of the sample and employed methodology in Section Methodology. Section Results presents the descriptive statistics and the result of the negative binomial regression. Finally, Section Discussion discusses the results in the context of the previous literature, and Section Conclusion provides concluding remarks along with the practical implications and future research directions.

\section{LITERATURE REVIEW}

\section{Fake News Ecosystem}

A fake news ecosystem refers to an online environment whereby users, who have different motivations and seek entertainment through interaction with others, reciprocate by creating and spreading fake news across different domains (Kim et al., 2019). Research has addressed many user-related factors that facilitate the spread of fake news. First and foremost, users are not likely to probe the news that is aligned with their existing beliefs and values due to confirmation bias (Kim et al., 2019; Lazer et al., 2018; Torres et al., 2018; Vasu et al., 2018). This is because they generally cannot resist psychological pleasure from engaging with this ideologically compatible news (Allcott \& Gentzkow, 2017; Bakshy et al., 2015; Spohr, 2017). The second reason is the users' reluctance to alter their attitudes in favor of a conflicting view, even after the fake news has been debunked (Marwick, 2018). These users deliberately ignore supporting evidence to avoid the mental discomfort incurred from acknowledging the opposing view's rightness. Even worse, it may lead to a backfire effect where users become even more motivated to share fake news when the evidence is indisputably presented (Flynn et al., 2017; Nyhan \& Reifler, 2010; Pennycook et al., 2020) or to a reduction of overall news consumption (Mitchell et al., 2019). Last, users do not use social media platforms to inform others. Instead, they share to signal their identities, values, and norms (Allcott \& Gentzkow, 2017; Marwick, 2018; Pennycook et al., 2020).

Noticing the commonalities in what most grabs users' attention on social media, the content generators structure their content in a way that attracts the utmost interaction with other users (Menninghaus et al., 2016; Mihailidis \& Viotty, 2017), hence securing higher financial returns (Enoch Pratt Free Library, n.d.; Tambini, 2017). In that sense, fake content circulating amongst social media users can be viewed as a digital meme, the smallest unit of cultural transmission and imitation (Dawkins, 2016). From this perspective, the chance of survival is directly dependent on its transmission rate (i.e., popularity amongst users). This survivalcentric interpretation is plausible given that only a tiny proportion of tweets can achieve a high level of popularity (i.e., transmission rate), indicated by retweets count, likes, and replies (Burkhardt, 2017). If content finds its way to a sufficient number of users who share it without detecting it as fake, it will go viral in just a few seconds (Hamidian \& Diab, 2019; Jin et al., 2017; Papanastasiou, 2020). This is the ultimate motivation of the generators in creating fake news.

The probability of fake content being viral also varies with the topic category of the content. While the spread of fake political news is accelerated by echo chambers in social media (Mihailidis \& Viotty, 2017), this may not be the case for fake news in the category of technology. As a matter of fact, users' criteria to construct 
source credibility can change (Hilligoss \& Rieh, 2008). Although previous research examined the differences between spreading patterns of fake news in various categories (e.g., Vosoughi et al., 2018), it failed to identify the content-related factors accounting for the variability of popularity levels. Therefore, we need to understand how generators' structure and differentiate their tweets to attain the highest possible popularity in each category.

In sum, past research has well established why users do not stop engaging with fake news. However, the degree to which content-related factors affect the popularity of fake news remains underexplored in the extant literature. Past research has mastered explaining social media users' political behaviors through the psychological phenomena mentioned above; however, studies that adopt a contextualized approach to explain online user behaviors in different topic categories are lacking. For instance, confirmation bias can explain why users continuously endorse ideologically aligned political fake news. However, it may not explain why the same user shares fake news in the science category. Therefore, the research should lend itself to discovering the topic-related factors that users consider in their engagement decisions.

\section{Understanding Fake News from a Rhetorical Perspective}

Like his many other writings, Aristotle's Rhetoric has a substantial impact on the whole field of rhetoric as an art. Mainly focusing on the speaker's ability to find the persuasive in every given case (Rapp,2010), Aristotle grouped the means of rhetoric under what we now call the rhetorical triangle: ethos, pathos, and logos. While the first component concerns the speaker's identity, the second is more about the nature of the audience and how to mobilize them, mainly through the arousal of emotions. The last component is directly related to the message itself a speaker attempts to communicate to an audience (Grant, 2019). The research has shown that this triangular formulation may cater to fake news research by systematically evaluating the contents (e.g., Burkhardt, 2017, Murungi et al., 2018). A possible mapping between the rhetorical triangle and the elements of a fake tweet matches the ethos with the credibility of the source who posted the fake tweets first, pathos with the emotional elements embedded in the tweet to receive reactions from other users, and logos with the content itself. This rhetorical approach thus provides a viable way to analyze fake tweets.

\section{Ethos}

The credibility of sources may influence the effect range of fake news. For example, suppose the source is a popular Twitter user with tens of thousands of followers. In that case, the range exponentially grows through the followers who do not doubt the post's authenticity shared by someone they think is trustworthy and competent (Fritch \& Cromwell, 2002). The personal influence theory posits that any media message is mediated by opinion leaders (Katz \& Lazarsfeld, 1966). The same effect applies to social media with a minor substitution of opinion leaders with influencers in social media terminology. We can, therefore, assert a strong role for ethos in the spread of fake news. The research has identified many user-related indicators that can relate to ethos: number of followers, number of followings, number of tweets, number of favorites, and whether the account is verified or not (Alrubaian et al., 2018; Indu \& Thampi, 2019; Jin et al., 2017). For example, a significant number of followers indicates the effect range of a particular user since it is the size of the audience who would see this user's post on their feeds (Burkhardt, 2017).

\section{Pathos}

Considering the nature of the "emotion-based" fake news market (Rochlin, 2017, p. 386], we can cast an important role for pathos that addresses emotional bonds with audiences for effectively communicating a message. Arousal of emotions and an emphasis on common beliefs and values are central themes of pathos. Its existence often manifests itself in demagogic statements with extreme polarities in tweets (Dochoff \& Hays, 2017; Grant, 2019). Prosodic tools such as emoticons are also common to reinforce the emotions and opinions embedded in the message (Lohmann et al., 2017). This component is particularly emphasized in political messages where the political figure seeks to build common ground with the voters (Grant, 2019). Fortunately, sophisticated methods are available to quantify the emotions and opinions embedded in a tweet. For instance, sentiment analysis is often used to extract polarity based on the expressed opinions and emotions in the text (Bondielli \& Marcelloni, 2019; Elmurngi \& Gherbi, 2017). Additionally, the polarity of emojis, the 


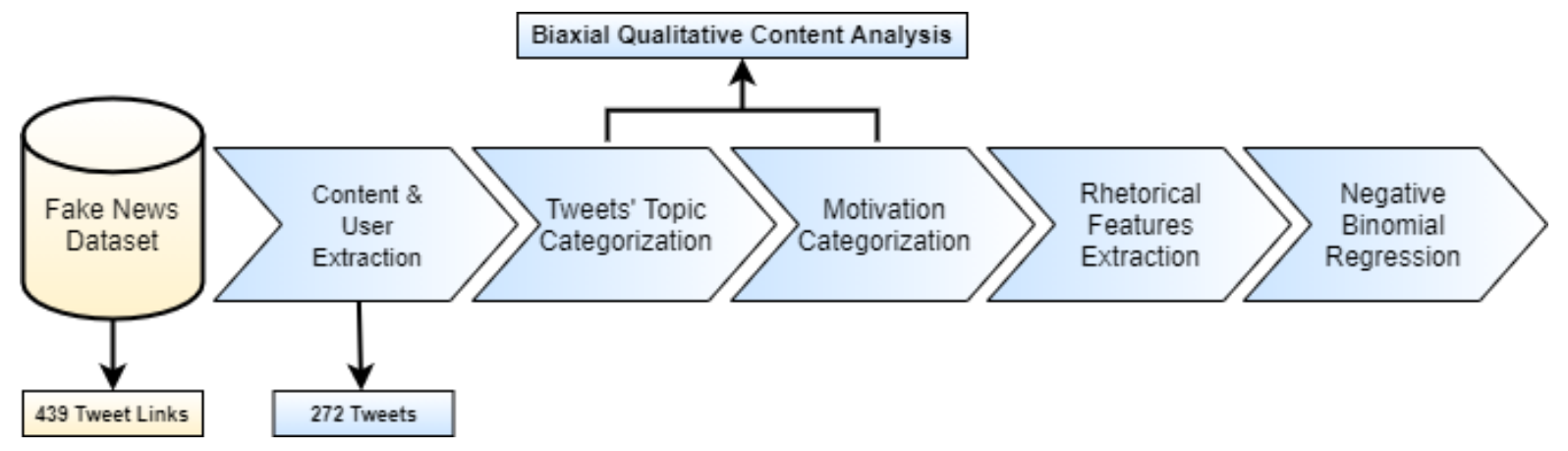

Figure 1. Methodology

presence of exclamation marks, and words in all capital letters (like yelling) in a tweet may also be used to reinforce the opinions and emotions stated in a tweet.

\section{Logos}

When fake news producers are now continuously improving their imitation abilities by neutralizing the language and removing grammatical errors from tweets to evade detection (Burkhardt 2017), social media users rely on a set of criteria for evaluating the logic of a message, including neutrality, specificity, and meaningfulness (Marcella et al., 2019). The logos can be quantified through many indicators. In practice, a precise, closed, and plain language message without any emotional elements may signify logos. The most straightforward approach for incorporating logos is to investigate the presence of common elements: several characters, many words, question marks, capital letters, punctuation, negations, first-person pronouns, and third-person pronouns (Jin et al., 2017; Ma et al., 2015; Yang et al., 2018). Apart from the elements of a traditional message, a tweet may contain some unique elements: hashtag, mention. Also, Twitter users can attach a photo, video, or link to their tweets. These elements should also be viewed as content-related features.

Rhetorical features that contribute to the spread of fake news on Twitter are the focal points of the current study. Unfortunately, previous research has failed to provide a precise mapping of indicators into relevant rhetorical components. Therefore, we will attempt to organize indicators that are frequently used to characterize fake news under relevant components.

\section{METHODOLOGY}

\section{Sample}

Fake news data were collected through an independent Turkish fact-checking organization known as teyit.org. This organization is a member of The International Fact-Checking Network and is fully committed to objectivity and openness, correction policy, and economic transparency (teyit, n.d.). There exists a body of research using datasets provided by teyit.org (Karadağ \& Ayten, 2020; Ünal \& Çiçeklioğlu, 2019). As shown in Figure 1, we obtained a sample, including 439 confirmed fake tweet links. We revisited each link and extracted category, design motivation, and rhetorical features from 272 available tweets.

\section{Biaxial Qualitative Content Analysis}

Qualitative content analysis is a systematic coding and categorization approach to discover and interpret large amounts of textual data (Iosifidis \& Nicoli, 2020). We created a biaxial coding scheme using an iterative process to identify 1) the tweet's topic or category and 2) the designer's motivation to spread them (Chew \& Eysenbach, 2010).

First, we defined our codes regarding the tweets' topics by conducting a preliminary analysis. Then, we gathered topics under the classification of soft news, hard news, and general news (Lehman-Wilzig \& Seletzky, 2010). For example, we classified news in politics, history, economy, and finance as hard news that requires instant reporting due to its significance and short lifetime. On the other hand, we included the news in life, celebrity, and sports under soft news that contains light or exotic topics. Soft news can be reported later or 
Table 1. Intercoder consistency

\begin{tabular}{lcccccc}
\hline & \multicolumn{5}{c}{ Topic Categories Design Motivations } \\
\hline & $1^{\text {st }}$ Round & $2^{\text {nd }}$ Round & $3^{\text {rd }}$ Round & $1^{\text {st }}$ Round & $2^{\text {nd }}$ Round & $3^{\text {rd }}$ round \\
\hline Judge 1-Judge 2 & $60.33 \%$ & $97.33 \%$ & $100.00 \%$ & $79.18 \%$ & $99.10 \%$ & $100.00 \%$ \\
Judge 1-Judge 3 & $71.48 \%$ & $90.83 \%$ & $100.00 \%$ & $49.67 \%$ & $90.91 \%$ & $100.00 \%$ \\
Judge 1-Judge 4 & $76.95 \%$ & $89.58 \%$ & $100.00 \%$ & $51.17 \%$ & $90.91 \%$ & $100.00 \%$ \\
Judge 2-Judge 3 & $69.53 \%$ & $97.41 \%$ & $100.00 \%$ & $81.19 \%$ & $100.00 \%$ & $100.00 \%$ \\
Judge 2-Judge 4 & $50.49 \%$ & $90.23 \%$ & $100.00 \%$ & $50.74 \%$ & $90.91 \%$ & $100.00 \%$ \\
Judge 3-Judge 4 & $51.71 \%$ & $91.48 \%$ & $100.00 \%$ & $51.19 \%$ & $90.91 \%$ & $100.00 \%$ \\
\hline
\end{tabular}

Note: The Kappa result between 0.81 and 1 indicates almost perfect agreement (McHugh, 2012).

even not all. Lastly, we involved news in science, technology, and health under the classification of general news that includes up-to-date news. However, it is not necessary to report them instantly.

In the first round, we worked independently. The coding unit was a single tweet, and we coded every single unit with only one category. In the second and third rounds, we resolved any disagreements in coding in additional meetings. We all discussed disagreements in detail, reached a mutual agreement, and changed the coding sheet as needed.

Second, we adopted a codebook from the literature to code the motivation to spread tweets consisting of fake news (Meel \& Vishwakarma, 2019). This codebook contained nine motives: political intent, financial profit, passion for promoting an ideology, fun, increasing customer base, rush to cover the latest news, generating advertising revenues, technological reasons, and manipulating public opinion (see Appendix A). In the first round, we again worked individually. The coding unit was a single tweet, and we coded every single unit with only one motive. Table 1 summarizes the intercoder consistencies between each pair of judges.

\section{Rhetorical Feature Extraction}

We operationalized the logos component of the rhetorical framework by encoding the tweets based on the presence of certain elements: photo, video, hashtag, mention, question mark, and ellipsis (see Appendix B). The pathos of a tweet was operationalized through three indicators: the presence of emoji and exclamation marks, the sum of sentiment score of a tweet, and emoji polarity. We used SentiStrength, a sentiment analysis tool (Thelwall et al., 2010) that uses a Turkish sentiment dictionary called Zemberek (Vural et al., 2013) to produce polarity scores between -5 and 5 . We obtained positive and negative sentiment scores for each tweet and summed them to calculate the overall polarity. The dominant type of emoji in a tweet may be assumed as the overall polarity. We categorized emoji polarity as negative (-1), neutral (0), positive (1), and none (2). For the ethos, we used account-level features. We extracted the number of followers, followings, tweets posted, tweets liked. Also, we examined whether the related account was verified or not. We used the Twitter API to extract these features.

The retweet count measures fake news popularity because only the original and retweeted posts are shown in users' feeds on Twitter. Therefore, we estimate the spreading rate with the retweet count instead of the number of likes or replies of a tweet. Table 2 summarizes the extracted features included in the current study. 
Table 2. Features

\begin{tabular}{|c|c|}
\hline Feature & Description \\
\hline Fake news popularity & The number of times a tweet is retweeted \\
\hline Category & The category of fake news \\
\hline Motivation & Design motivation of a tweet \\
\hline \multicolumn{2}{|l|}{ Rhetorical Features } \\
\hline \multicolumn{2}{|l|}{ Logos } \\
\hline Photo & Whether it contains a photo \\
\hline Video & Whether it contains a video \\
\hline Link & Whether it contains a link \\
\hline Hashtag & Whether it contains a hashtag \\
\hline Mention & Whether it contains a mention \\
\hline Question Mark & Whether it contains a question mark \\
\hline Ellipsis & Whether it contains more than one dot \\
\hline \multicolumn{2}{|l|}{ Pathos } \\
\hline Sentiment Score & Total polarity score of a message \\
\hline Emoji Polarity & Total emoji polarity \\
\hline Exclamation Mark & Whether it contains an exclamation mark \\
\hline All Capital & Whether all text is in capital letters \\
\hline \multicolumn{2}{|l|}{ Ethos } \\
\hline \# followers & Number of followers \\
\hline \# followings & Number of following \\
\hline \# tweets & Number of tweets \\
\hline \# favorites & Number of favorites \\
\hline Verification status & Whether account is verified or not \\
\hline
\end{tabular}

\section{Negative Binomial Regression}

Negative binomial Poisson is well-suited for predicting over-dispersed count data (Ver Hoef \& Boveng, 2007). This model's advantage is its ability to model Poisson heterogeneity when the variance is not equal to the mean. (For further reading, please see Cameron \& Trivedi, 2013). The formula for negative binomial regression is:

$$
P\left(Y_{i}=y_{i}\right)=\frac{\Gamma\left(y_{i}+\phi\right)}{y_{i} ! \Gamma(\phi)} \frac{\phi^{\phi} \lambda_{i}^{y_{i}}}{\left(\phi+\lambda_{i}\right)^{\phi-y_{i}}}
$$

$\Gamma$ is a gamma function and is the reciprocal of the residual variance of the underlying mean. The natural logarithm for the number of the expected number of events is formulated as:

$$
\ln \left(\lambda_{i}\right)=\sum_{k=0}^{K} \beta_{k} x_{i k}
$$

where $x_{i k}$ is the independent variables $x$ and is the estimated coefficient.

The negative binomial regression also allows us to infer the significance and magnitude of the main effects of various tweet characteristics on the retweet counts.

\section{RESULTS}

\section{Descriptive Statistics}

Table 3 summarizes the frequencies of each topic category and design motivations. Table 3 identified 140 tweets as hard news, 115 as soft news, and only 17 as general news. Moreover, Table 3 indicates that Twitter users mostly posted this fake content because of political intent $(n=69)$. The results also indicated that users mainly wanted to manipulate public opinion $(n=64)$ and increase their customer base $(n=60)$ by gaining readership. Furthermore, users preferred to post fake news because of the rush to cover the latest news, passion for promoting an ideology, and fun. However, we could not find any content posted to generate financial profit, advertising revenues, and technological reasons. Additionally, Table 4 presents the descriptive statistics of rhetorical features across logos, pathos, and ethos. 
Table 3. Descriptive statistics of contextual features

\begin{tabular}{lcc}
\hline Contextual Features & Frequency & Percentage (\%) \\
\hline Category & & \\
Hard news (C1) & 140 & 51.5 \\
Soft news (C2) & 115 & 42.3 \\
General news (C3) & 17 & 6.2 \\
Motivation & 69 & 25.4 \\
Political intent (M1) & 0 & 0 \\
Financial profit (M2) & 21 & 7.6 \\
Passion for promoting an ideology (M3) & 12 & 4.3 \\
Fun (M4) & 60 & 21.8 \\
Increase customer base (M5) & 46 & 17 \\
Rush to cover the latest news (M6) & 0 & 0 \\
Generate advertising revenues (M7) & 0 & 0 \\
Technological reasons (M8) & 64 & 23.9 \\
Manipulate public opinion (M9) & & \\
\hline
\end{tabular}

Table 4. Descriptive statistics of rhetorical features

\begin{tabular}{|c|c|c|c|c|}
\hline Rhetorical Features & $\mathrm{N}$ & Percentage (\%) & Mean & SD \\
\hline \multicolumn{5}{|l|}{ Logos } \\
\hline Photo & $188(84)$ & $69(31)$ & & \\
\hline Video & $54(218)$ & $20(80)$ & & \\
\hline Link & $18(254)$ & $7(93)$ & & \\
\hline Hashtag & $66(206)$ & $24(76)$ & & \\
\hline Question mark & $29(243)$ & $11(89)$ & & \\
\hline Ellipsis & 208(64) & $26(74)$ & & \\
\hline Mention & $9(263)$ & $3(97)$ & & \\
\hline \multicolumn{5}{|l|}{ Pathos } \\
\hline Sentiment Score & & & -0.27 & 1.280 \\
\hline \multicolumn{5}{|l|}{ Emoji Polarity } \\
\hline Negative & 15 & 4.4 & & \\
\hline Neutral & 25 & 9.2 & & \\
\hline Positive & 15 & 5.5 & & \\
\hline None & 220 & 80.9 & & \\
\hline Exclamation mark & $52(220)$ & $19(81)$ & & \\
\hline All capital & $12(260)$ & $96(4)$ & & \\
\hline \multicolumn{5}{|l|}{ Ethos } \\
\hline \# followers & & & 602,600 & $23,151,253.5$ \\
\hline \# followings & & & $28,036.30$ & $154,939.9$ \\
\hline \# tweets & & & $58,129.31$ & $110,859.25$ \\
\hline \# favorites & & & $27,508.41$ & $51,348.508$ \\
\hline Verification Status & $80(192)$ & $29(71)$ & & \\
\hline
\end{tabular}

The numbers in the parentheses refer to the number and percentage of tweets without the specified element

\section{Negative Binomial Regression}

The model's main effects results, presented in Table 5, suggested significant differences between both topic and motivation categories. For example, while hard news was 2.09 times more likely to be retweeted, this rate was 3.00 times greater than soft news than general news. Regarding design motivations, fake news created with the motivation of a rush to cover the latest news was nearly 4.00 times less likely to be retweeted concerning manipulation of public opinion. There was no significant difference for any of the remaining categories in the current sample other than manipulating the public opinion category. 
Table 5. Negative binomial regression results-main effects

\begin{tabular}{|c|c|c|c|}
\hline \multirow[t]{2}{*}{ Model Comparison } & \multicolumn{3}{|c|}{ Main Effects } \\
\hline & B & SE & $\operatorname{Exp}(B)$ \\
\hline Intercept & $4.61^{* *}$ & 1.00 & 101.2 \\
\hline \multicolumn{4}{|l|}{ Category } \\
\hline C1 & $0.73^{*}$ & 0.36 & 2.09 \\
\hline C2 & $1.10^{* *}$ & 0.32 & 3.00 \\
\hline$C 3^{\mathrm{a}}$ & - & - & - \\
\hline \multicolumn{4}{|l|}{ Motivation } \\
\hline M1 & -0.64 & 0.20 & 0.93 \\
\hline M3 & 0.22 & 0.27 & 0.41 \\
\hline M4 & 0.01 & 0.37 & 1.01 \\
\hline M5 & 0.07 & 0.24 & 1.07 \\
\hline M6 & $-1.31^{* *}$ & 0.23 & 0.26 \\
\hline $\mathrm{M9}^{\mathrm{a}}$ & - & - & - \\
\hline \multicolumn{4}{|l|}{ Logos } \\
\hline Photo & 0,38 & 0,29 & 1,46 \\
\hline Video & 0.16 & 0.32 & 1.17 \\
\hline Hashtag & -0.15 & 0.21 & 0.86 \\
\hline Question mark & 0.06 & 0.23 & 1.07 \\
\hline Ellipsis & -0.27 & 0.16 & 0.75 \\
\hline Mention & $1.11^{* *}$ & 0.39 & 3.056 \\
\hline \multicolumn{4}{|l|}{ Pathos } \\
\hline Sentiment score & -0.41 & 0.05 & 0.960 \\
\hline \multicolumn{4}{|l|}{ Emoji Polarity } \\
\hline Negative & $-1.35^{* *}$ & 0.49 & 0.259 \\
\hline Neutral & -0.05 & 0.54 & 0.943 \\
\hline Positive & $-1.18^{* *}$ & 0.44 & 0.307 \\
\hline None $^{a}$ & - & - & - \\
\hline Exclamation mark & $0.91^{* *}$ & 0.17 & 2.484 \\
\hline All capital & 0.14 & 0.33 & 1.160 \\
\hline \multicolumn{4}{|l|}{ Ethos } \\
\hline \# followers & $0 * * 1$ & 0 & 1 \\
\hline \#followings & 0 & 0 & 1 \\
\hline \# tweets & $0 * * 2$ & 0 & 1 \\
\hline \# favorites & 0 & 0 & 1 \\
\hline Verification Status & 0.03 & 0.16 & 1.03 \\
\hline
\end{tabular}

Note: ** significant at $a=.05$ level, $* * *$ significant at $a=.01$ level $a=$ reference category

${ }^{1}$ The coefficient is too small, yet it has a significantly positive impact on popularity $\left(B=1.44 * 10^{-7}\right)$

2 The coefficient is too small, yet it has a significantly negative impact on popularity $\left(B=-2.17^{\star} 10^{-6}\right)$

Mention was the single significant predictor of popularity with regards to the logos of a tweet. Fake tweets without mention were 3.05 times more likely to be retweeted. Retweet counts for tweets with negative emojis were nearly 4.00 times less compared to the tweets without any emojis.

Additionally, the counts of tweets without an exclamation mark were 2.50 times greater than those with an exclamation mark. Thus, while the number of followers was significantly related to popularity, the relationship was statistically negative for the number of tweets.

Furthermore, incorporating interaction effects produced differential relationships between rhetorical features, motivations, and categories (See Table 6). Interestingly, the hard news category suggests that fake tweets' popularity without an exclamation mark is negative $\left(B=-29.72, S E=0.71, \operatorname{Exp}(B)=1.175^{\star} 10^{-13}\right)$ compared to the fake news with an exclamation mark. The same relationship also holds for the question mark, where fake tweets without a question mark were 35.62 times more likely to be retweeted in this category. Popularity of fake news from non-verified accounts was significantly higher regarding hard news $(B=5.78, S E=1.43, \operatorname{Exp}(B)=326.49)$ and soft news $(B=3.49, S E=1.31, \operatorname{Exp}(B)=32.93)$. However, its impact is nonsignificant regarding general news. The number of those following the source was also a significant predictor of popularity regarding hard news and soft news. The hashtag was found to decrease the popularity of fake hard news in the contents created with a political intent $(B=1.54, S E=0.54, E x p(B)=4.67)$. Mentions increase the popularity of fake tweets used for spreading an ideology $(B=-4.92, S E=2.37, \operatorname{Exp}(B)=0.007)$. 
Table 6. Negative binomial regression results-interaction effects

\begin{tabular}{|c|c|c|c|}
\hline \multirow[t]{2}{*}{ Model Comparison } & \multicolumn{3}{|c|}{ Significant Interaction Effects } \\
\hline & $B$ & SE & $\operatorname{Exp}(B)$ \\
\hline $\mathrm{C} 1 *$ Exclamation Mark $=0$ & $-29.72^{* *}$ & 0.71 & $0^{4}$ \\
\hline $\mathrm{C}{ }^{*}$ Question Mark $=0$ & $3.56^{* *}$ & 0.32 & 35.62 \\
\hline C1*All Capital $=0$ & $7.49^{*}$ & 3.22 & 1797.40 \\
\hline $\mathrm{C}_{1} *$ Verified Account $=0$ & $3.49^{* *}$ & 1.31 & 32.98 \\
\hline$C 2 *$ Verified Account $=0$ & 0.22 & 0.27 & 0.41 \\
\hline $\mathrm{C} 1 *$ Number of Followings $=0$ & 1 & 1 & 1.00 \\
\hline $\mathrm{C} 2{ }^{*}$ Number of Followings $=0$ & $2^{*}$ & $2^{*}$ & 1.00 \\
\hline M6*Photo $=0$ & $2.27^{*}$ & 1.15 & 9.70 \\
\hline M3* Link $=0$ & $12.62^{* *}$ & 2.69 & 302459 \\
\hline M1*Hashtag $=0$ & $1.54^{*}$ & 0.70 & 4.67 \\
\hline M3*Mention $=0$ & $-4.92^{*}$ & 2.37 & 0.007 \\
\hline M5*Emoji Polarity=-1 & $3^{*}$ & 3 & 3 \\
\hline M1*All capital $=0$ & $-7.00^{*}$ & 3.10 & 0.01 \\
\hline M1*Number of Followers & $4^{* *}$ & 4 & 4 \\
\hline M6* Number of Tweets & $5^{* *}$ & 5 & 5 \\
\hline M6* Number of Followings & $6^{* *}$ & 6 & 6 \\
\hline M6* Number of Favorites & $6^{* *}$ & 6 & 6 \\
\hline M5*Polarity & $-0.74^{*}$ & 0.34 & 0.473 \\
\hline
\end{tabular}

Note: ** significant at $a=.05$ level, *** significant at $\alpha=.01$ level $a=$ reference category

${ }^{1}$ The coefficient is too small, yet it has a significantly positive impact on popularity $\left(B=7.43^{*} 10^{-5}\right)$

${ }^{2}$ The coefficient is too small, yet it has a significantly negative impact on popularity $\left(B=7.12^{*} 10^{-5}\right)$

${ }^{3}$ The coefficient is too small, yet it has a significantly negative impact on popularity $\left(B=1.13^{*} 10^{-6}\right)$

${ }^{4}$ The coefficient is too small, yet it has a significantly negative impact on popularity $\left(B=-2.19 * 10^{-6}\right)$

${ }^{5}$ The coefficient is too small, yet it has a significantly negative impact on popularity $\left(B=-1.40 * 10^{-5}\right)$

${ }^{6}$ The coefficient is too small, yet it has a significantly positive impact on popularity $\left(B=3.14^{*} 10^{-5}\right)$

Similarly, all capital letters in fake tweets used for political intent significantly increased the popularity. Polarity was found to affect popularity in the fifth motivation category negatively. The number of favorites of the source was positively related to the popularity of tweets used for the rush to cover the latest news category. The number of those following the source and the number of tweets were negatively related to popularity in the same motivation category. The number of followers was negatively affected by the popularity of a fake tweet used for political intents.

\section{DISCUSSION}

The results confirmed the role of the topic category in the popularity of fake news. We found that people were more likely to share fake hard news and soft news than general news. This result paralleled past research (Nielsen \& Graves, 2017; Vosoughi et al., 2018), indicating popularity differences across fake news categories. The possible explanation could be people's natural attraction to politics and celebrity topics. For example, stating an opinion about a political issue signals orientation and identity, confirming the need to relate with others (Allcott \& Gentzkow, 2017; Marwick, 2018; Pennycook et al., 2020). From the uses and gratification theory, the third category's unpopularity can be explained by the fact that social media users are less likely to engage with informational content (Cvijikj \& Michahelles, 2013; Lee et al., 2013) compared to relational or entertainment contents, which could loosely be mapped to first and second categories, respectively.

We found that fake news created with the motivation of manipulating public opinion received much more interaction from users than a rush to cover the latest news did. A possible explanation would be that time pressure for covering a story about the latest news may result in a speculative but haphazard structure. This structure might curtail believability and, thus, engagement with this news (Kim et al., 2019). The results confirm the number of fabrication and sophistication efforts to increase the popularity (Vosoughi et al., 2018), which is less likely to exist in inelaborate content.

This study addressed the effects of rhetorical features on the spread of fake news. For example, in the pathos category, positive or negative emojis and exclamation marks surprisingly rendered a tweet less popular. These results indicate that the current sample responded to emotional elements in a negative way. 
It implies that users are skeptical about the authenticity of content emphasizing emotions, showing a particular media literacy level (Burkhardt, 2017). Related to the credibility of the source-ethos- we found significant positive and negative effects on the number of followers and tweets on fake news's popularity, respectively. It suggested that the effect range of fake news is magnified by the source's popularity, highlighting social media influencers' great responsibility (Bovet \& Makse, 2019).

The findings empirically supported the interaction between rhetorical features and category and motivation. For example, fake tweets without a question mark or all capital letters were found to be more prevalent in the category of hard news. One striking interaction effect is that verification status and the number of followed persons negatively affect popularity. However, their impacts were found insignificant in the category of general news. These results implied that users paid more attention to a sign of authenticity and the source's popularity when they interact with informational content. In sum, all the findings supported that content popularity differed across categories and motivations (Vosoughi et al., 2018).

In short, the proposed multistep approach combining biaxial content analysis and negative binomial regression to a large extent confirmed the proposed fake news market analogy. The sellers' (i.e., fake news generators) have products sold at different rates across different sections (i.e., categories) by emphasizing certain features (i.e., rhetorical features). This context-aware understanding of fake news helps better characterize and identify the fake news plaguing the online communication channels in various domains. The proposed methodology also addresses fake news from the creators' perspective. It thus provides valuable insights about possible tricks embedded in fake content in each category.

\section{CONCLUSION}

Our study showcased the primary impacts of topic and design motivation categories and rhetorical features on the popularity of fake tweets. The main results uncovered the effects of rhetorical features on the popularity of a tweet. Much to our surprise, the existence of pathos-related features decreased, rather than increased, the number of retweets of fake content. In contrast, the credibility of sources significantly increased the popularity of fake news. Further results empirically supported the differential impacts of rhetorical features on popularity across topic and motivation categories. It indicates the evident differences in the fake tweets in different categories. Identifying the salient characteristics of fake tweets in topic and motivation categories undoubtedly helps quickly spot fake news.

This study has many practical implications. First and foremost, this study supplements automated factchecking efforts with a contextualized rhetorical approach that immediately identifies the risk elements of fake news. This rapid characterization could help develop a system that automates the labeling process and thus enables early intervention (Papanastasiou, 2020). Given that labeling fake news could sometimes take up to three days (Silverman, 2017), developing such systems warrants huge public safety and health benefits. For instance, Twitter has lately introduced an internal system that puts automatic labels on the disputed and misleading contents related to COVID-19 (Roth \& Pickles, 2020). Although the initial results are quite promising, the platform still relies on some trusted partners to identify fake content. Second, accurately identifying the peculiar characteristics across topic categories and design motivations allows the development of a risk score that indicates the degree of the fakeness of content. For example, an excessive emphasis on emotions contributes to that risk score in the hard news category. These scores can then be used to rank contents across each category and trigger an early intervention for the contents with the highest risk before they find their ways to be shared by many users. Lastly, the proposed marketplace analogy provides valuable insights into how fake news survives in an ecosystem. A social media literacy program that capitalizes on these insights can inform users about how fake content generators with various motivations structure their contents to target their cognitive weaknesses. Having equipped with the necessary information, users can develop a contextualized evaluation skills about the credibility of the posts they are about to share.

The research contributed to the extant literature by showing how the rhetorical framework could be used as a systematic way of evaluating content under three components: ethos, pathos, and logos (Murungi et al., 2018). This framework might help researchers identify influential factors on popularity and characterize fake news from different perspectives (Burkhardt, 2017). It also shows the necessity to delve into more contextualized relationships regarding the popularity of fake news. 
Our study has some limitations. First, we applied the negative binomial regression on relatively small sample size. Second, we had to use the dataset from a single fact-checking organization, which does not allow generalizability. The proposed framework should, therefore, be replicated with different samples. Third, we limited our sample only to tweets to explore the underlying dynamics of fake news popularity. Finally, the same approach should be applied to other social media platforms or news sites by considering the changing user characteristics of different social media platforms.

In the future, we plan to work on more massive datasets, including different fake news sources. We also consider a cross-country study to reveal cultural and linguistic differences by replicating the same analysis under different study settings. A methodological triangulation with a qualitative analysis to identify the factors that fake content generators consider represents another exciting direction for the future study.

Author contributions: All authors were involved in concept, design, collection of data, interpretation, writing, and critically revising the article. All authors approve final version of the article.

Funding: The authors received no financial support for the research and/or authorship of this article.

Declaration of interest: Authors declare no competing interest.

Data availability: Data generated or analysed during this study are available from the authors on request.

\section{REFERENCES}

Allcott, H., \& Gentzkow, M. (2017). Social media and fake news in the 2016 election. Journal of Economic Perspectives, 31(2), 211-236. https://doi.org/10.1257/jep.31.2.211

Alrubaian, M., Al-Qurishi, M., Hassan, M. M., \& Alamri, A. (2018). A credibility analysis system for assessing information on Twitter. IEEE Transactions on Dependable and Secure Computing, 15(4), 661-674. https://doi.org/10.1109/TDSC.2016.2602338

Bakshy, E., Messing, S., \& Adamic, L. A. (2015). Exposure to ideologically diverse news and opinion on Facebook. Science, 348(6239), 1130-1132. https://doi.org/10.1126/science.aaa1160

Barthel, M., Mitchell, A., \& Holcomb, J. (2016). Many Americans believe fake news is sowing confusion. Pew Research Center: Journalism \& Media. https://www.journalism.org/2016/12/15/many-americansbelieve-fake-news-is-sowing-confusion/

Bondielli, A., \& Marcelloni, F. (2019). A survey on fake news and rumour detection techniques. Information Sciences, 497, 38-55. https://doi.org/10.1016/j.ins.2019.05.035

Bovet, A., \& Makse, H. A. (2019). Influence of fake news in Twitter during the 2016 U.S. presidential election. Nature Communications, 10. https://doi.org/10.1038/s41467-018-07761-2

Burkhardt, J. M. (2017). Combating fake news in the digital age. American Library Association.

Cameron, A. C., \& Trivedi, P. K. (2013). Regression analysis of count data (2nd Ed.). Cambridge University Press.

Chew, C., \& Eysenbach, G. (2010). Pandemics in the age of Twitter: Content analysis of Tweets during the 2009 H1N1 outbreak. PLOS ONE, 5(11), e14118. https://doi.org/10.1371/journal.pone.0014118

Cvijikj, I. P., \& Michahelles, F. (2013). Online engagement factors on Facebook brand pages. Social Network Analysis and Mining, 3(4), 843-861. https://doi.org/10.1007/s13278-013-0098-8

Dawkins, R. (2016). The selfish gene. Oxford University Press.

Dochoff, C. H., \& Hays, M. (2017). Fake furnishings: real and fake reporting on white house remodeling. http://publish.illinois.edu/dochoff2/files/2017/04/article-1-revised.pdf

Elmurngi, E., \& Gherbi, A. (2017). Detecting fake reviews through sentiment analysis using machine learning techniques. In S. Bhulai, \& D. Kardaras (Eds), Proceedings of the Sixth International Conference on Data Analytics (DATA ANALYTICS 2017) (pp. 65-72). International Academy, Research, and Industry Association (IARIA).

Enoch Pratt Free Library (n.d.). Fake news: How to spot it. https://www.prattlibrary.org/research/tools/index.aspx?cat=90\&id=4735

Flynn, D. J., Nyhan, B., \& Reifler, J. (2017). The nature and origins of misperceptions: Understanding false and unsupported beliefs about politics. Political Psychology, 38(S1), 127-150. https://doi.org/10.1111/pops.12394

Fritch, J. W., \& Cromwell, R. L. (2002). Delving deeper into evaluation: exploring cognitive authority on the Internet. Reference Services Review, 30(3), 242-254. 
Grant, A. J. (2019). Ethos, pathos and logos: rhetorical fixes for an old problem: Fake news. In SITE 2019: Informing Science + Information Technology Education Conference (pp. 81-91). Informing Science Institute. https://doi.org/10.28945/4154

Hamidian, S., \& Diab, M. T. (2019). Rumor detection and classification for Twitter data. arXiv.org. https://arxiv.org/ftp/arxiv/papers/1912/1912.08926.pdf

Hilligoss, B., \& Rieh, S. Y. (2008). Developing a unifying framework of credibility assessment: Construct, heuristics, and interaction in context. Information Processing \& Management, 44(4), 1467-1484. https://doi.org/10.1016/j.ipm.2007.10.001

Indu, V., \& Thampi, S. M. (2019). A nature-inspired approach based on Forest Fire model for modeling rumor propagation in social networks. Journal of Network and Computer Applications, 125, 28-41. https://doi.org/10.1016/j.jnca.2018.10.003

Iosifidis, P., \& Nicoli, N. (2020). The battle to end fake news: A qualitative content analysis of Facebook announcements on how it combats disinformation. International Communication Gazette, 82(1), 60-81. https://doi.org/10.1177/1748048519880729

Ipsos Public Affairs. (2019). CIGI Ipsos global survey: Internet security \& trust 2019 part 3: Social media, fake news \& algorithms. https://www.cigionline.org/sites/default/files/documents/2019\%20CIGI-Ipsos\%20Global \%20Survey\%20-\%20Part\%203\%20Social\%20Media\%2C\%20Fake\%20News\%20\%26\%20Algorithms.pdf

Jin, Z., Cao, J., Guo, H., Zhang, Y., \& Luo, J. (2017). Multimodal fusion with recurrent neural networks for rumor detection on microblogs. Proceedings of the 25th ACM International Conference on Multimedia (pp. 795816). Association for Computing Machinery (ACM).

Karadağ, G. H., \& Ayten, A. (2020). A comparative study of verification/fact-checking organzaitons in Turkey: dogrulukpayi.com and teyit.org. Motif Akademi Halkbilimi Dergisi, 13(29), 483-501. https://doi.org/10.12981/mahder.673262

Katz, E., \& Lazarsfeld, P. F. (1966). Personal influence: The part played by people in the flow of mass communications. Transaction Publishers.

Kim, A., Moravec, P. L., \& Dennis, A. R. (2019). Combating fake news on social media with source ratings: The effects of user and expert reputation ratings. Journal of Management Information Systems, 36(3), 931-968. https://doi.org/10.1080/07421222.2019.1628921

Lazer, D. M., Baum, M., Benkler, Y., Berinsky, A. J., Greenhill, K. M., Menczer, F., Metzger, M. J., Nyhan, B., Pennycook, G., Rothschild, D., Schudson, M., Sloman, S. A., Sunstein, C., Thorson, E. A., Watts, D. J., \& Zittrain, J. L. (2018). The science of fake news. Science, 359(6380), 1094-1096. https://doi.org/10.1126/science.aao2998

Lee, D., Hosanagar, K., \& Nair, H. S. (2013). The effect of advertising content on consumer engagement: Evidence from Facebook. http://www.researchgate.net/publication/257409065

Lehman-Wilzig, S. N., \& Seletzky, M. (2010). Hard news, soft news, 'general' news: The necessity and utility of an intermediate classification. Journalism, 11(1), 37-56. https://doi.org/10.1177/1464884909350642

Levine, S., \& Gambino, L. (2021, February 14). Donald Trump acquitted in second impeachment trial. The Guardian. https://www.theguardian.com/us-news/2021/feb/13/donald-trump-acquitted-impeachmenttrial

Lohmann, K., Pyka, S. S., \& Zanger, C. (2017). The effects of smileys on receivers' emotions. Journal of Consumer Marketing, 34(7), 489-495. https://doi.org/10.1108/JCM-02-2017-2120

Ma, J., Gao, W., Wei, Z., Lu, Y., \& Wong, K. F. (2015). Detect rumors using time series of social context information on microblogging websites. Proceedings of the 24th ACM International on Conference on Information and Knowledge Management (pp. 1751-1754). https://doi.org/10.1145/2806416.2806607

Marcella, R., Baxter, G., \& Walicka, A. (2019). User engagement with political "facts" in the context of the fake news phenomenon: an exploration of information behaviour. Journal of Documentation, 75(3), 10821099. https://doi.org/10.1108/JD-11-2018-0180

Marwick, A. E. (2018). Why do people share fake news? A sociotechnical model of media effects. Georgetown Law Technology Review, 2(2), 474-512.

McHugh, M. L. (2012). Interrater reliability: The kappa statistic. Biochemia Medica, 22(3), 276-282. https://doi.org/10.11613/B.M.2012.031 PMID:23092060 
Meel, P., \& Vishwakarma, D. K. (2019). Fake news, rumor, information pollution in social media and web: A contemporary survey of state-of-the-arts, challenges and opportunities. Expert Systems with Applications, 153. https://doi.org/10.1016/j.eswa.2019.112986

Menninghaus, W, Bohrn, I. C., Knoop, C. A., Kotz, S. A., Schlotz, W., \& Jacobs, A. M. (2016). Rhetorical features facilitate prosodic processing while handicapping ease of semantic comprehension. Cognition, 143, 4860. https://doi.org/10.1016/j.cognition.2015.05.026

Mercier, H. 2020. Fake news in the time of coronavirus: How big is the threat? The Guardian. https://www.theguardian.com/commentisfree/2020/mar/30/fake-news-coronavirus-false-information

Mihailidis, P., \& Viotty, S. (2017). Spreadable spectacle in digital culture: Civic expression, fake news, and the role of media literacies in "post-fact" society. American Behavioral Scientist, 61(4), 441-454. https://doi.org/10.1177/0002764217701217

Mitchell, A., Gottfried, J., Stocking, G., Walker, M., \& Fedeli, S. (2019). Many Americans say made-up news is a critical problem that needs to be fixed. Pew Research Center Journalism \& Media. https://www.journalism.org/2019/06/05/many-americans-say-made-up-news-is-a-critical-problem-thatneeds-to-be-fixed/

Mitchell, A., Simmons, K., Matsa, K. E., \& Silver, L. (2018). Publics globally want unbiased news coverage, but are divided on whether their news media deliver. Pew Research Center Global Attitudes \& Trends. https://www.pewresearch.org/global/2018/01/11/publics-globally-want-unbiased-news-coverage-butare-divided-on-whether-their-news-media-deliver/

Murungi, D., Purao, S., \& Yates, D. (2018). Beyond facts: a new spin on fake news in the age of social media. Twenty-fourth Americas Conference on Information Systems (pp. 1-10). Association for Information Systems (AIS).

Nielsen, R. K., \& Graves, L. (2017). “News you don't believe": Audience perspectives on fake news. Reuters Institute for the Study of Journalism. https://reutersinstitute.politics.ox.ac.uk/our-research/news-you-dontbelieve-audience-perspectives-fake-news

Nyhan, B., \& Reifler, J. (2010). When corrections fail: The persistence of political misperceptions. Political Behavior, 32(2), 303-330. https://doi.org/10.1007/s11109-010-9112-2

Papanastasiou, Y. (2020). Fake news propagation and detection: A sequential model. Management Science, 66(5), 1826-1846. https://doi.org/10.1287/mnsc.2019.3295

Pennycook, G., Bear, A., Collins, E. T., \& Rand, D. G. (2020). The implied truth effect: Attaching warnings to a subset of fake news headlines increases perceived accuracy of headlines without warnings. Management Science, 66(11), 4944-4957. https://doi.org/10.1287/mnsc.2019.3478

Rapp, C. (2002). Aristotle's rhetoric. The Stanford Encyclopedia of Philosophy (Spring 2010 Edition), Edward N. Zalta (ed.). https://plato.stanford.edu/archives/spr2010/entries/aristotle-rhetoric

Rochlin, N. (2017). Fake news: Belief in post-truth. Library Hi Tech, 35(2), 386-392. https://doi.org/10.1108/LHT03-2017-0062

Roth, Y., \& Pickles, N. (2020). Updating our approach to misleading information. https://blog.twitter.com/en_us/topics/product/2020/updating-our-approach-to-misleading-information

Silverman, B. (2017). Facebook says its fact checking program helps reduce the spread of a fake story by $80 \%$. Buzzfeed News. https://www.buzzfeednews.com/article/craigsilverman/facebook-just-shared-the-firstdata-about-how-effective-its

Spohr, D. (2017). Fake news and ideological polarization: Filter bubbles and selective exposure on social media. Business Information Review, 34(3), 150-160. https://doi.org/10.1177/0266382117722446

Tambini, D. (2017). Fake news: Public policy responses. Media Policy Brief 20. London School of Economics and Political Science. https://core.ac.uk/download/pdf/80787497.pdf

teyit. Methodology: teyit. https://teyit.org/methodology/

Thelwall, M., Buckley, K., Paltoglou, G., Cai, D., Kappas, A. (2010). Sentiment strength detection in short informal text. Journal of the American Society for Information Science and Technology, 61(12), 2544-2558. https://doi.org/10.1002/asi.21416

Torres, R., Gerhart, N., \& Negahban, A. (2018). Epistemology in the era of fake news: An exploration of information verification behaviors among social networking site users. ACM SIGMIS Database, 49(3), 7897. https://doi.org/10.1145/3242734.3242740 
Ünal, R., \& Çiçeklioğlu, A. Ş. (2019). The function and importance of fact-checking organizations in the era of fake news: teyit.org, an example from Turkey. Media Studies, 10(19), 140-160. https://doi.org/10.20901/ms.10.19.8

Vasu, N., Ang, B.,,Teo, T. A., Jayakumar, S., Raizal, M., \& Ahuja, J. (2018). Fake news: national security in the posttruth era. Policy Report. Global Resilience Institute at Northeastern University. https://www.rsis.edu.sg/wp-content/uploads/2018/01/PR180313_Fake-News_WEB.pdf

Ver Hoef, J. M., \& Boveng, P. L. (2007). Quasi-Poisson vs. negative binomial regression: How should we model overdispersed count data? Ecology, 88(11), 2766-2772. https://doi.org/10.1890/07-0043.1

Vosoughi, S., Roy, D., \& Aral, S. (2018). The spread of true and false news online. Science, 359(6380), 1146-1151. https://doi.org/10.1126/science.aap9559

Vural, A. G., Cambazoglu, B. B., Senkul, P., \& Tokgoz, Z. O. (2013). A framework for sentiment analysis in Turkish: Application to polarity detection of movie reviews in Turkish. In E. Gelenbe, \& R. Lent (Eds), Computer and information sciences III. Springer. https://doi.org/10.1007/978-1-4471-4594-3_45

Wardle, C., \& Derakhshan, H. (2017). Information disorder: Toward an interdisciplinary framework for research and policy making. Report Ref. 162317GBR. Council of Europe.

West, D. (2021). The role of misinformation in Trump's insurrection. Brookings. https://www.brookings.edu/ blog/techtank/2021/01/11/the-role-of-misinformation-in-trumps-insurrection/

Wise, A. (2021). Acting capitol police chief promises 'significant' changes following deadly riot. NPR. https://www.npr.org/sections/insurrection-at-the-capitol/2021/02/05/964616916/acting-capitol-policechief-promises-significant-changes-following-deadly-riot

Yang, Y., Zheng, L., Zhang, J., Cui, Q., Li, Z., \& Yu, P. S. (2018). TI-CNN: Convolutional neural networks for fake news detection. arXiv. https://arxiv.org/abs/1806.00749

Zannettou, S., Sirivianos, M., Blackburn, J., \& Kourtellis, N. (2019). The web of false information: Rumors, fake news, hoaxes, clickbait, and various other shenanigans. Journal of Data and Information Quality, 11(3), 137. https://doi.org/10.1145/3309699 
E. Akar et al.

\section{APPENDIX A}

\section{The Codebook of Motivations to Spread Fake Tweets}

We adopted this codebook from Meel and Vishwakarma (2019).

\begin{tabular}{ll}
\hline Motive & Description \\
\hline Political Intent & $\begin{array}{l}\text { People post fake news to damage the public image of an opponent or support a } \\
\text { political figure or party }\end{array}$ \\
$\begin{array}{l}\text { Financial Profit } \\
\text { Passion for promoting an } \\
\text { ideology }\end{array}$ & $\begin{array}{l}\text { People, who are impassioned about an organization, person, ideology, or } \\
\text { philosophy want to disseminate it and post about it even if it is fake. }\end{array}$ \\
$\begin{array}{l}\text { Fun } \\
\text { Increase Customer Base }\end{array}$ & $\begin{array}{l}\text { People post fake news to have fun, although it is not harmful. } \\
\text { Online news media post content without questioning it to increase their customer }\end{array}$ \\
Rush to cover the latest news & $\begin{array}{l}\text { Journalists post content without fact-checking to cover the story first and get } \\
\text { millions of views. }\end{array}$ \\
$\begin{array}{l}\text { Generate advertising } \\
\text { revenues }\end{array}$ & $\begin{array}{l}\text { Fake news authors make money from automated advertising engines such as } \\
\text { Technological reasons }\end{array}$ \\
$\begin{array}{l}\text { Facebook Ads and Google Adsense. } \\
\text { Search engine algorithms promote fake news due to its popularity and intentional } \\
\text { design to gain more users. }\end{array}$ \\
$\begin{array}{l}\text { Customers decide the future of stocks, sales, election results, business, and more } \\
\text { so that their public opinion is significantly vital for customers. }\end{array}$ \\
\hline
\end{tabular}




\section{APPENDIX B}

\section{Twitter Features}

The first tweet is written in all caps and includes an ellipsis, exclamation mark, a video, and a link.

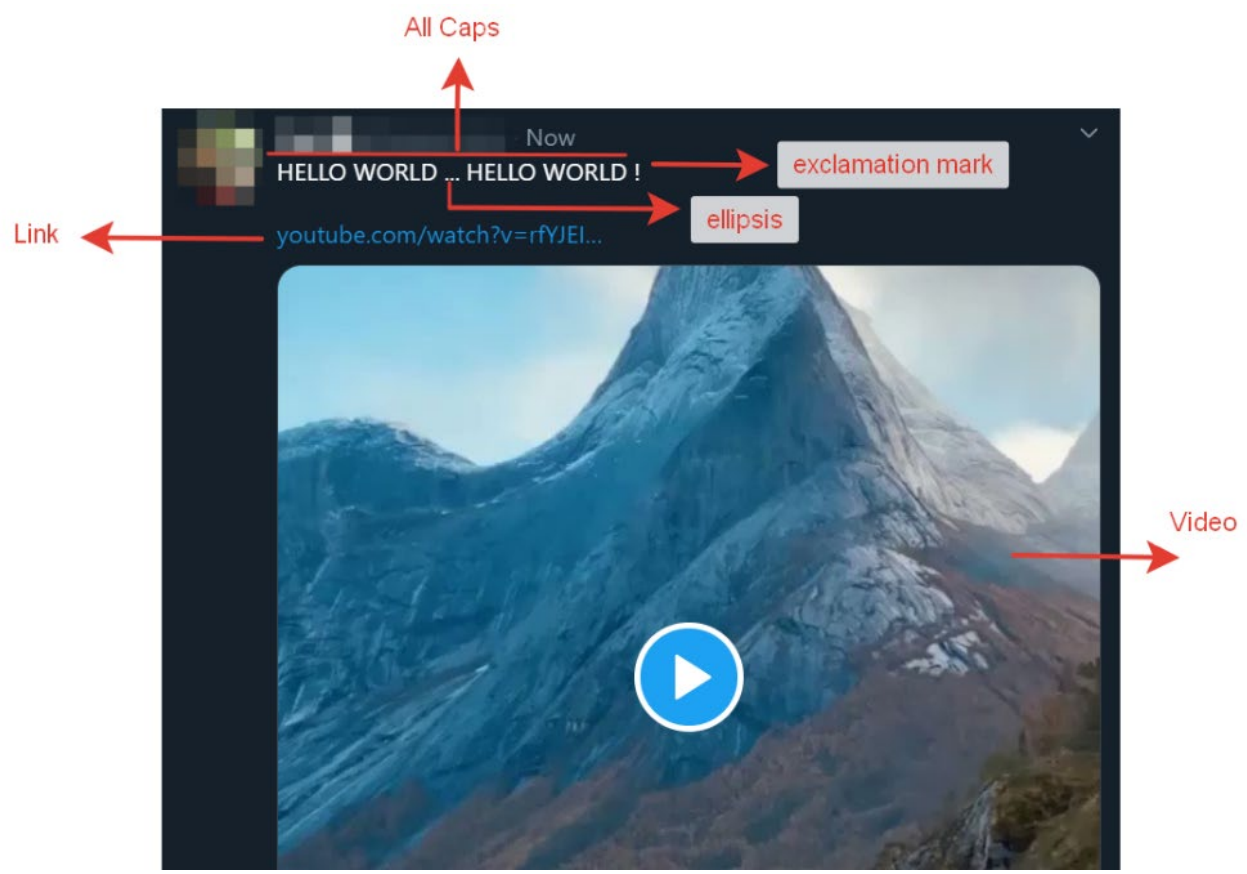

The second tweet includes a hashtag, mention, picture, and emoji.
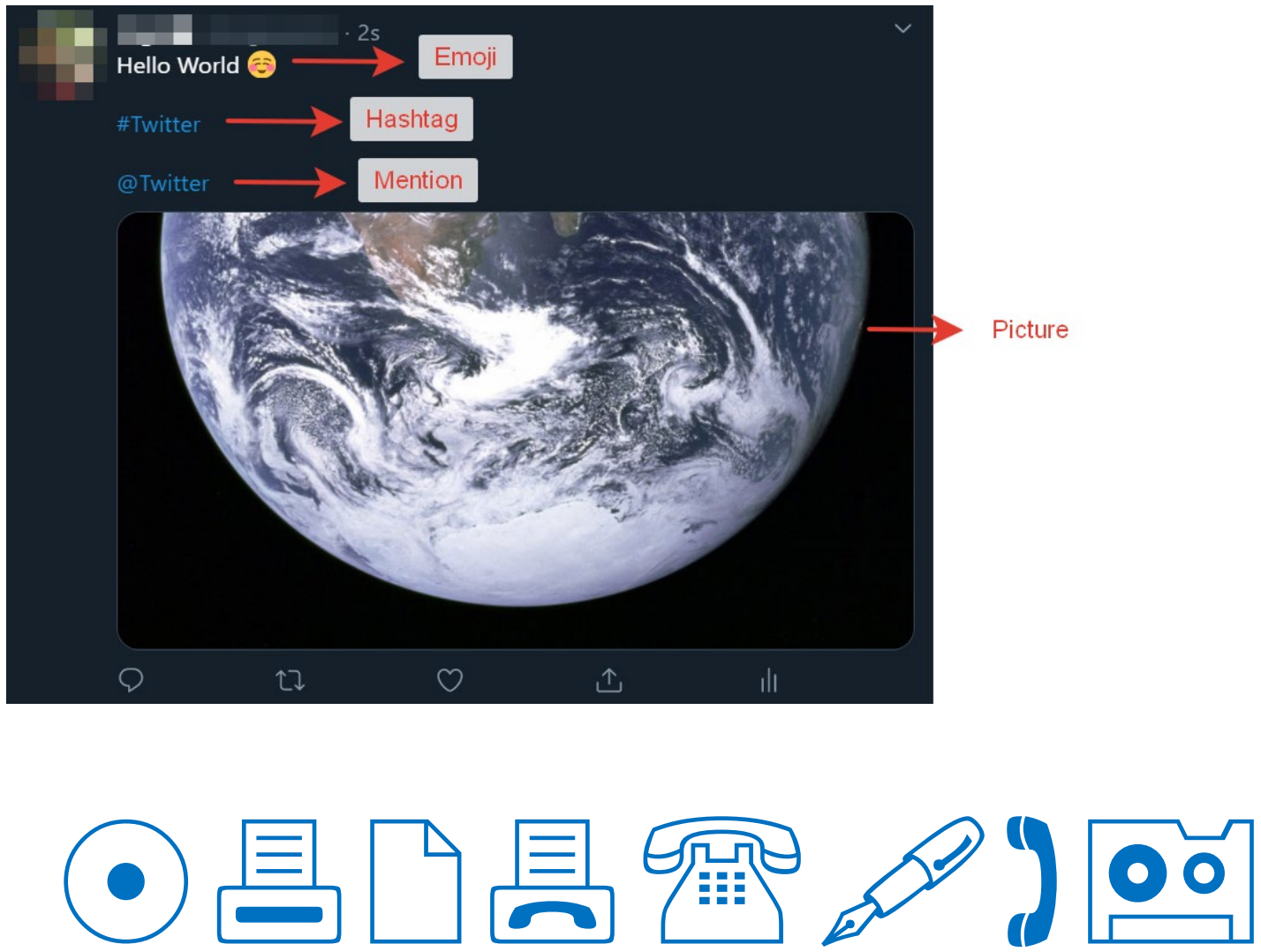